ISSN 1991-8631

Original Paper

http://indexmedicus.afro.who.int

\title{
Diversité des systèmes d'élevages de bovins de race bovine Borgou dans la zone soudanienne du Bénin
}

\author{
Issaka YOUSSAO ABDOU KARIM ${ }^{1 *}$, M. DAHOUDA $^{2}$, E .Y. ATTAKPA ${ }^{3}$, \\ G.B. KOUTINHOUIN ${ }^{1}$, G.S. AHOUNOU ${ }^{1}$, S.S. TOLEBA ${ }^{2}$ et B.S. BALOGOUN ${ }^{1}$ \\ ${ }^{1}$ Université d'Abomey-Calavi, Ecole Polytechnique d'Abomey-Calavi, Département de Production et \\ Santé Animales, 01 BP 2009 Cotonou, Bénin. \\ ${ }^{2}$ Université d'Abomey-Calavi, Faculté des Sciences Agronomiques, Département de Production Animales, \\ 01 BP 526 Cotonou, Bénin. \\ ${ }^{3}$ Faculté d'Agronomie de Parakou / Université de Parakou, B.P. 123, Parakou, Bénin. \\ *Auteur Correspondant, E-mail : iyoussao@yahoo.fr, issaka.youssao@epac.uac.bj,BP 2009 Cotonou, Bénin. \\ Tél : 0022995285988 ou 00229979120 74, Fax : 0022921360199
}

\section{RESUME}

La caractérisation des systèmes d'élevage permet d'identifier la diversité des élevages afin d'élaborer des actions de développement. Le but de cette étude est de caractériser les types d'élevages de bovins de race Borgou dans la zone septentrionale du Bénin. La collecte des données a eu lieu dans 40 élevages dans l'Alibori, 24 dans l'Atacora et 84 dans le Borgou, de juin à décembre 2010. La procédure Proc corresp a été utilisée pour l'analyse factorielle des correspondances (AFC). Les Peulh représentent l'ethnie majoritaire des éleveurs (62,5 à 95,8\%). Les élevages de type 1 se rencontrent dans les Communes de Pèrèrè, de Banikoara et chez un groupe d'éleveurs de Tchaourou. Ils sont des éleveurs professionnels ou des agriculteurs et font le plus souvent un élevage de prestige. Ils sont le plus souvent des Peulh ou des Gando. Dans le type 2, les éleveurs de bovins de Kèrou et du second groupe d'éleveurs de Tchaourou sont des éleveurs professionnels et secondairement des agriculteurs. Ils sont pour la plupart Peulh. Ils vendent du lait, des animaux de trait et des bovins de boucherie élevés en embouche herbagère sur pâturage naturel. Le type 3 concerne les éleveurs de bovins de Parakou qui ont la boucherie ou le commerce comme activité principale et qui produisent de la viande à travers l'engraissement des animaux. Ils sont Bariba ou Dendi. La caractérisation des trois types d'élevage permettra de proposer des actions de développement intégrées.

() 2013 International Formulae Group. All rights reserved.

Mots clés: Alimentation, économie, habitat, pathologie, système d'élevage.

\section{INTRODUCTION}

Le cheptel national bovin est composé d'une diversité génétique constituée de races bovines Lagunaire, Somba, Borgou et des zébus, principalement, zébu Peul, White Fulani et M'Bororo Rouge. Certaines races ont été importées et élevées dans les Fermes d'Etat : il s'agit de la race N'Dama à la Ferme d'Elevage de l'Okpara et des Girolando à la Ferme d'Elevage de Kpinnou. Il existe enfin des métis issus de croisements entre diverses races bovines. Parmi ces races, la race Borgou 
issue d'un croisement stabilisé entre les zébus, principalement White Fulani et la race taurine Somba, occupe $51 \%$ du cheptel bovin national (MAEP, 2007). Originaire du Département du Borgou (Bénin) dont il porte le nom, son aire de distribution géographique s'étend dans les Départements de l'Alibori, de la Donga, des Collines et du Zou. Son aire de distribution s'étend au Nigeria (Kétéku), au Burkina Faso (Méré) et au Togo (Youssao et al., 2009). Son mode d'élevage est de type extensif et traditionnel (sédentaire ou transhumant), basé sur l'exploitation du pâturage naturel. Elle possède une trypanotolérance au même titre que les races Lagunaire et N'Dama (Doko et al., 2010). Selon Domingo (1976), la robe dominante de la race Borgou est blanche ou grise, parfois pie noire, les extrémités sont généralement noires (mufle, vulve, creux de l'oreille, sourcil et sabot). Reconnue comme une race adaptée du Nord Bénin, la race Borgou est utilisée pour trois fins : traction animale (culture et transport), lait et viande. De nombreux travaux scientifiques ont été réalisés pour caractériser et améliorer les performances zootechniques de cette race (Youssao et al., 2000a, 2000b; Adamou N'Diaye et al., 2001 ; Youssao et al., 2007, 2009 ; Koutinhouin et al., 2009). La plupart de ces travaux ont été réalisés dans les fermes d'Etat ou dans les projets de développement où le mode d'élevage est de type semi amélioré. Malgré l'apport de ces différents résultats sur l'amélioration de la productivité de la race Borgou, le développement de l'élevage bovin dans les élevages traditionnels doit passer d'abord par une caractérisation des pratiques d'élevages. Face à l'extrême diversité des situations à décrire, les typologies ont l'ambition de constituer un jeu de types qui simplifie la réalité tout en respectant les particularités principales (Perrot et Landais, 1993; Djenontin et al., 2004 ; Alkoiret et al., 2009). Les typologies d'exploitations agricoles permettent de comparer des groupes d'exploitations entre elles, de juger de leur fonctionnement, d'identifier des solutions éventuelles aux problèmes rencontrés et d'élaborer des recommandations adaptées (Perrot et Landais 1993; Djenontin et al., 2004; Alkoiret et al., 2009). L'objectif des typologies est donc de fournir à l'usage des décideurs une image de l'activité agricole locale pour orienter les actions de développement (Roybin, 1987).

Une enquête a été réalisée par Alkoiret et al. (2009) sur la typologie des élevages bovins de la Commune de Gogounou. Cette étude se limite à une des Communes productrices de lait qui vient en troisième position en termes d'effectif après Banikoara et Kalalé (CeRPA, 2009). L'objectif de cette étude est de caractériser les types d'élevage de bovins de race Borgou pratiqués dans son berceau, notamment dans la zone septentrionale du Bénin.

\section{MATÉRIEL ET MÉTHODES Milieu d'étude}

La collecte des données dans le cadre de cette étude, a été réalisée simultanément dans les Départements de l'Alibori, de l'Atacora et du Borgou (Figure 1).

Le Département de l'Alibori est situé dans l'extrême Nord du Bénin entre $10^{\circ} 49^{\prime}$ $60^{\prime \prime}$ et $11.86^{\circ}$ latitude Nord et $2^{\circ} 25^{\prime} 60^{\prime \prime}$ et $3^{\circ}$ 41' 40" longitude Est. Il a une superficie de $26242 \mathrm{~km}^{2}$ (23\% du territoire national) et une population estimée à 521093 habitants. Le climat et la végétation sont de type soudanosahélien avec une saison de pluie (mai à septembre) et une saison sèche (novembre à avril). La pluviométrie varie entre 700 et 1000 mm (Adam et Boko, 1993).

Le Département de l'Atacora est situé à l'extrême Nord-Ouest du Bénin entre $9^{\circ} 62^{\prime} 0^{\prime \prime}$ et $10^{\circ} 49^{\prime} 30^{\prime \prime}$ latitude Nord et $1^{\circ} 23^{\prime} 19^{\prime \prime}$ et $2^{\circ}$ 6' 34" longitude Est. Sa superficie est de $20499 \mathrm{~km}^{2}$. Le nombre d'habitants de ce département est de 549417 habitants avec une densité de 27 habitants par $\mathrm{km}^{2}$. Le Département de l'Atacora est doté d'un climat 
de type soudanien, marqué par deux saisons : une saison pluvieuse d'avril à octobre et une saison sèche de novembre à mars. En outre, la moyenne de précipitations est élevée dans cette région (1300 mm par an) et celle-ci est liée au climat de montagne qui y sévit (Adam et Boko, 1993).

Le Département du Borgou est situé au Nord-Est du Bénin entre $8^{\circ} 52^{\prime} 60^{\prime \prime}$ et $10^{\circ} 25^{\prime}$ $60^{\prime \prime}$ latitude Nord et $2^{\circ} 36^{\prime} 0^{\prime \prime}$ et $3^{\circ} 41^{\prime} 40^{\prime \prime}$ longitude Est. Il couvre une superficie de $25856 \mathrm{~km}^{2}$ dont $13962 \mathrm{~km}^{2}$ de terres cultivables, soit $54 \%$ de la superficie totale du département. Le climat est de type soudanien avec en alternance une saison pluvieuse (mai à octobre) et une saison sèche (novembre à avril) où l'harmattan souffle entre décembre et février. La pluviosité moyenne est de 1200 mm (Adam et Boko, 1993).

\section{Matériel}

Le matériel utilisé est composé d'une fiche d'enquête et des bovins de race Borgou. La fiche d'enquête renferme les informations suivantes: l'identification des éleveurs, la constitution des troupeaux, la description de l'habitat, la taille et la structure du troupeau, l'alimentation, le suivi sanitaire, l'utilisation des animaux et de leurs productions et le mode de financement.

\section{Méthode d'enquête}

La méthodologie utilisée pour la collecte des données est celle de l'enquête rétrospective par entretien avec l'éleveur. Cette enquête a permis de recueillir aussi bien les informations sur l'éleveur que sur les caractéristiques de son élevage. Les éleveurs ont été choisis suivant les critères d'accessibilité et leur disponibilité à fournir les informations. La collecte des données a eu lieu dans 40 élevages dans l'Alibori, 24 dans l'Atacora et 84 dans le Borgou, de juin à décembre 2010.

\section{Analyse statistique}

Les données collectées sur le terrain ont été codées et enregistrées dans une base de données conçue sur le logiciel Excel et analysées avec le logiciel SAS (1996). Pour les variables quantitatives (âge, superficie cultivée, effectifs des animaux par classe d'âge, taille des troupeaux), une analyse de variance à un seul facteur a été utilisée et le département où les enquêtes ont été réalisées a été la seule source de variation. La procédure Proc GLM a été utilisée pour l'analyse de variance. Les comparaisons entre les moyennes ont été faites deux à deux par le test de t. Les fréquences ont été calculées par la procédure Proc freq du SAS (1996) et comparées par le test de Chi-carré et par le test bilatéral de Z. Pour chaque fréquence relative, un intervalle de confiance (IC) à 95\% a été calculé suivant la formule :

$I P=1,96 \sqrt{\frac{[P(1-P)]}{N}}$

où $\mathrm{P}$ est la fréquence relative et $\mathrm{N}$ la taille de l'échantillon.

La procédure Proc corresp a été utilisée pour l'analyse factorielle des correspondances (AFC). Les variables qui ont été prises en compte étaient: département de l'éleveur, statut du propriétaire des animaux, destination des bovins et leurs produits (vente de bovin, vente de lait, culture attelée, production du fromage, autoconsommation du lait), modalités de vente (âge de l'animal, poids de l'animal, pathologie, période de fête, besoin d'argent), objectif de production (lait, viande, culture attelée ou le prestige), suivi des animaux (le propriétaire, son épouse, un employé) à plein temps ou à mi-temps, mode de traitement (vétérinaire ou pharmacopée) et chargé du suivi sanitaire des animaux (vétérinaire ou éleveur). Une classification ascendante hiérarchique sur la base des coordonnées des éleveurs sur les composantes de l'AFC les plus significatives a été ensuite réalisée. 


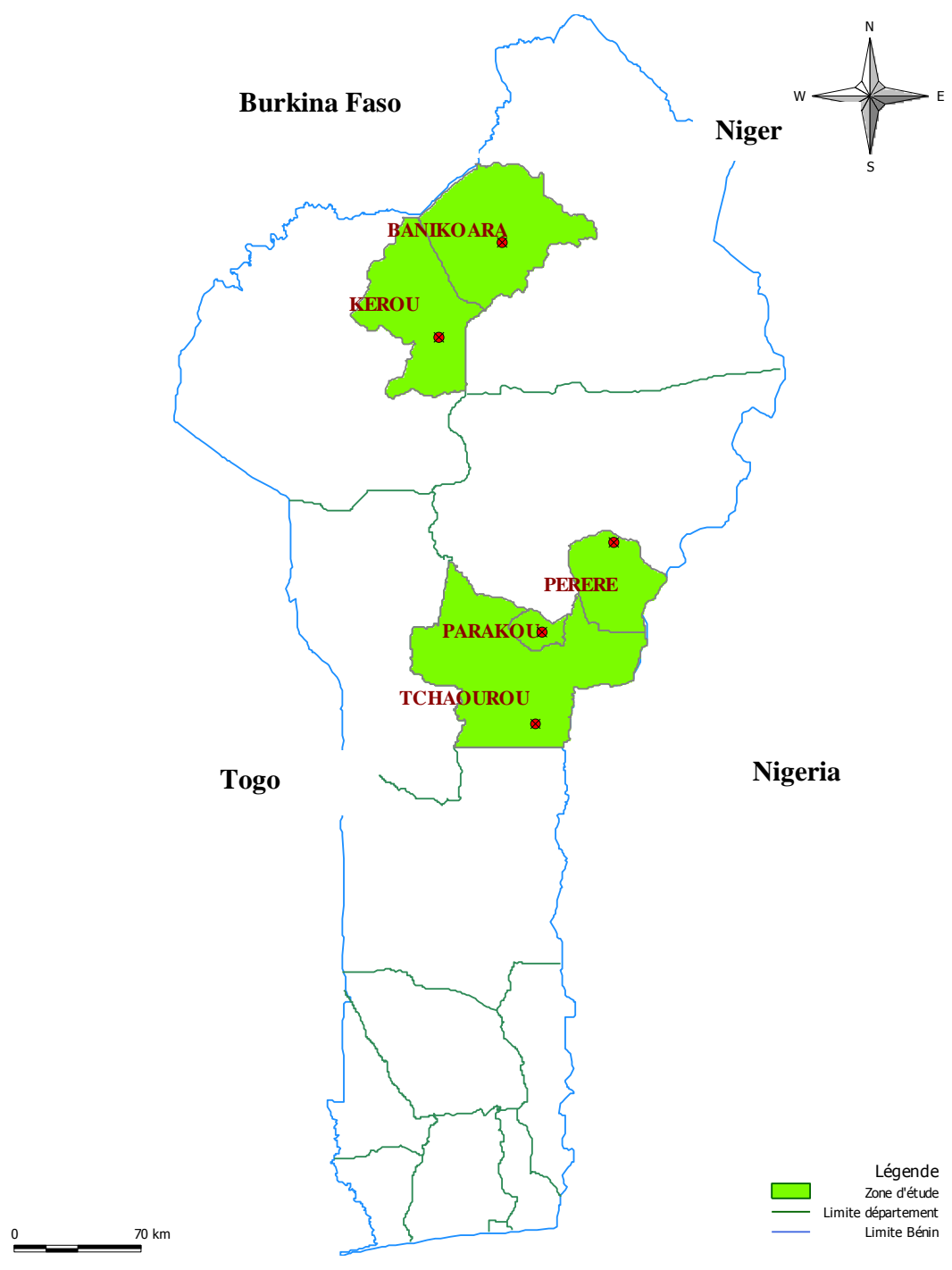

Océan Atlantique

Figure 1: Zone d'étude.

\section{RESULTATS}

\section{Statut de l'éleveur}

La proportion des différentes ethnies des éleveurs rencontrés dans ces trois Départements présente une différence significative $\quad(\mathrm{P}<0,001)$. Les éleveurs rencontrés dans le Département de l'Alibori étaient majoritairement Peulh $(62,5 \%)$, les Bariba (35\%) représentaient la seconde ethnie, venaient enfin les Gando (2,5\%). Dans le Département de l'Atacora, 95,83\% des éleveurs étaient Peulh et $4,17 \%$ étaient d'origine Djerma. Le Département du Borgou présente une diversité d'ethnies. Les Peulh étaient majoritaires et représentaient $74,12 \%$ des éleveurs. Les Gando (15,29\%), les Bariba $(7,06 \%)$, les Dendi $(2,35 \%)$ et les Somba $(1,18 \%)$ constituaient les minorités. En 
somme, il y avait plus d'éleveurs Peulh dans l'Atacora que dans le Borgou et dans l'Alibori $(\mathrm{P}<0,05)$ tandis que, les éleveurs Bariba étaient majoritaires dans l'Alibori que dans l'Atacora et le Borgou. Par rapport aux autres Départements, les éleveurs Gando ont été plus rencontrés dans le Borgou $(\mathrm{P}<0,05)$.

Les éleveurs des Départements de l'Atacora et du Borgou étaient exclusivement des hommes tandis que $97,5 \%$ des éleveurs du Département de l'Alibori étaient des hommes et $2,5 \%$ des femmes. Tous les éleveurs enquêtés dans l'Alibori et l'Atacora étaient mariés. Par contre dans le Borgou, 98,82\% des éleveurs enquêtés étaient mariés et 1,18\% étaient célibataires.

La religion islamique était pratiquée dans les trois Départements avec les proportions de $100,85,9$ et $67,5 \%$, respectivement dans l'Atacora, le Borgou et l'Alibori. Dans le Borgou, le christianisme a été la seconde religion $(14,12 \%)$. Par contre dans l'Alibori, les animistes et les athées, avec $12,5 \%$ chacun, représentaient la deuxième religion. Les éleveurs qui pratiquaient le christianisme dans l'Alibori ne représentaient que 5\% des enquêtés. La plupart des éleveurs rencontrés dans les trois départements (Atacora, Borgou et Alibori) étaient non scolarisés et représentaient respectivement 100, 93 et $92 \%$ des éleveurs interrogés $(\mathrm{P}>0,05)$. Seuls $7,9 \%$ et $7,1 \%$ des éleveurs rencontrés respectivement dans l'Alibori et dans le Borgou avaient un niveau secondaire.

L'âge moyen des éleveurs de bovins de race Borgou n'a pas été significativement différent en fonction des régions. Il était en moyenne de 47 ans dans l'Alibori, 41 ans dans l'Atacora et de 46 ans dans le Borgou. Le nombre de personnes à charge par éleveur a été similaire pour les trois régions $(\mathrm{P}<0,05)$. Dans l'Alibori, chaque éleveur avait en moyenne à sa charge 14 personnes, dans l'Atacora 13 personnes et dans le Borgou 16 personnes.

\section{Activités principales et secondaire des éleveurs}

Les activités principales pratiquées par les enquêtés étaient très peu diversifiées. L'élevage a été l'activité principale dominante dans les trois Départements. La proportion des éleveurs ayant pour activité principale l'élevage des bovins a été plus importante ( $\mathrm{P}<0,01)$ dans l'Atacora $(95,83 \%)$ que dans le Borgou (62,3\%) et dans l'Alibori (62,5\%). La seconde activité principale la plus pratiquée dans l'Alibori (35\%) et dans le Borgou $(29,41 \%)$ était l'agriculture. Les éleveurs qui menaient le commerce comme activité principale étaient présents dans les trois régions mais, avec des proportions similaires et faibles variant entre $2,35 \%$ et $4,17 \%$ (P>0,05). L'artisanat, la fonction publique, la boucherie et les conducteurs de véhicule constituaient les autres activités principales minoritaires enregistrées dans le Borgou avec des proportions respectives de $2,35 \%$; $1,18 \% ; 1,18 \%$ et $1,18 \%$ des éleveurs interrogés.

\section{Constitution des troupeaux}

Le don a été le premier mode d'acquisition des animaux dans l'Alibori $(94,74 \%)$ et dans l'Atacora $(95,83 \%)$, alors que dans le Borgou, il venait en seconde position $(38,82 \% ; \mathrm{P}<0,05)$. Par contre, la proportion des éleveurs qui ont constitué leur troupeau par achat a été majoritaire dans le Borgou $(74,12 \%)$ alors qu'elle n'était que de $13,16 \%$ dans l'Alibori et $4,17 \%$ dans l'Atacora. Dans le Borgou, la constitution du cheptel se réalisait aussi à travers l'héritage $(52,94 \%)$, l'échange $(37,65 \%)$, le confiage $(29,41 \%)$ et l'achat à crédit $(3,53 \%)$, et dans l'Alibori, à travers le confiage $(13,16 \%)$ et l'achat à crédit (10,53\%).

Le financement de l'élevage était assuré par trois acteurs : la famille, l'éleveur et les appuis extérieurs. Dans l'Atacora, $95,83 \%$ des éleveurs enquêtés ont reçu de l'aide de leur famille contre $81,18 \%$ dans le Borgou et $66,67 \%$ dans l'Alibori $(\mathrm{P}<0,05)$. 
Les proportions des éleveurs qui ont assuré eux-mêmes le financement de leur élevage dans l'Alibori $(69,23 \%)$ et le Borgou $(62,35 \%)$ ont été similaires et significativement plus importantes $(\mathrm{P}<0,05)$ que celle enregistrée dans l'Atacora (4,17\%). Aucune différence significative n'a été notée entre les proportions des éleveurs qui ont bénéficié des appuis extérieurs dans les trois régions.

\section{Structure du troupeau}

Les troupeaux rencontrés dans les trois zones d'étude présentaient des effectifs moyens en vaches, vaches allaitantes, veaux, velles, génisses 1 (1 à 2 ans), taurillons 1 (1 à 2 ans), genisses 2 (2 à 3 ans), taurillons 2 (2 à 3 ans), taureaux, bœufs, bouvillons 1 et bouvillons 2 similaires (Tableau 1). Par contre, les troupeaux rencontrés dans l'Atacora renfermaient des effectifs moyens en vaches gestantes et en vaches non gestantes significativement plus importants $(\mathrm{P}<0,001)$ que ceux enregistrés dans le Borgou et dans l'Alibori.

\section{Caractéristiques des habitats}

La totalité des enquêtés des trois Départements a utilisé le bois pour la construction des parcs de nuit. La moitié des éleveurs enquêtés attachaient les animaux sur des piquets dans les parcs de nuit par une corde dans l'Alibori, alors que tous les éleveurs le faisaient dans le Borgou et aucun éleveur ne le faisait dans l'Atacora $(\mathrm{P}<0,001)$.

\section{Suivi des animaux}

Les propriétaires qui suivaient en plein temps leurs animaux sont plus nombreux $(\mathrm{P}<0,05)$ dans l'Atacora $(58,33 \%)$ que dans le Borgou (34,12\%) et l'Alibori (12,5\%). La même tendance a été observée par rapport au suivi partiel des élevages par les propriétaires. Toutes les femmes des éleveurs de l'Atacora suivaient les animaux à mi-temps contre $20 \%$ de femmes dans l'Alibori et 5,88\% dans le Borgou $(\mathrm{P}<0,001)$. Le suivi à temps plein a été assuré par $21,18 \%$ des femmes des éleveurs dans le Borgou, tandis que dans
l'Alibori l'aide partielle a été pratiquée par $5 \%$ des femmes. Aucun enfant des éleveurs ne s'occupait du suivi des animaux dans l'Atacora. Dans l'Alibori, les enfants s'occupaient soit à plein temps $(2,5 \%)$, soit à mi-temps $(2,5 \%)$ ou soit partiellement $(5 \%)$ des animaux; alors que dans le Borgou, les enfants s'occupaient à mi-temps $(5,88 \%)$ des animaux. Les employés bouviers étaient surtout utilisés à plein temps dans l'Alibori (25\%) et dans le Borgou (49\%), alors que dans l'Atacora, ils étaient utilisés aussi bien à mi-temps (21\%) qu'à plein temps (25\%). Enfin, l'utilisation à mi-temps des employés a été réalisée par $1,18 \%$ des éleveurs du Borgou. Le suivi des animaux par les frères à l'éleveur a été pratiqué uniquement dans le Borgou par 10,59\% des éleveurs.

\section{Objectifs de production}

Les motivations des éleveurs à s'intéresser aux bovins étaient diversifiées. Il s'agit de la production des animaux de trait, du prestige, de la production de viande, de la production de lait, de la production de viande et de lait avec des fréquences qui ont varié d'un département à l'autre (Tableau 2). Contrairement aux autres Départements, aucun éleveur de l'Atacora n'élevait les animaux ni pour le prestige, ni pour la production $\mathrm{du}$ lait uniquement ou la production de viande uniquement, mais ils font tous la production de bovins à la fois pour le lait et la viande (Tableau 2).

La proportion des éleveurs qui vendent les bovins dans l'Atacora $(100 \%)$ et dans le Borgou $(98,82 \%)$ ne présentait pas de différence significative. Par contre, les fréquences de ces deux départements ont été plus importantes $(\mathrm{P}<0,05)$ que celle de l'Alibori (72,50\%). Les éleveurs de l'Atacora (100\%) qui vendaient du lait ont été plus importants $(\mathrm{P}<0,05)$ que ceux de l'Alibori (75\%) et du Borgou (76,47\%). La même tendance a été observée pour l'auto consommation du lait. La totalité des enquêtés de l'Atacora pratiquaient la culture attelée contre $77,5 \%$ dans l'Alibori et seulement $3,53 \%$ dans le Borgou $(\mathrm{P}<0,001)$ La 
proportion des enquêtés qui produisaient du fromage dans l'Atacora $(95,8 \%)$ a été significativement plus importante $(\mathrm{P}<0,05)$ que celles du Borgou (75,3\%) et de l'Alibori $(37,5 \%)$.

Les critères qui soutendent la vente des animaux ont été sensiblement les mêmes dans les trois Départements mais avec des proportions différentes (Tableau 2). Il s'agit de l'âge des animaux, les périodes de fêtes et le besoin d'argent. La totalité des éleveurs de l'Atacora tient compte du poids des animaux pour décider de leur vente contre $32,94 \%$ dans le Borgou $(\mathrm{P}<0,01)$ et ce critère n'a pas été pris en compte dans l'Alibori. La proportion des éleveurs qui vendaient les animaux en cas de pathologie ne présentait pas de différence significative entre l'Atacora $(41,67 \%)$ et le Borgou $(30,59 \%)$. La vente pour la culture attelée n'a été rencontrée que dans l'Alibori $(2,86 \%)$. La cause fondamentale de vente des animaux dans les trois Départements était la recherche de l'argent. Le déstockage, les pathologies, le renouvellement du cheptel ont été aussi les causes de vente des animaux, mais leurs proportions étaient faibles dans les trois Départements (Tableau 2).

\section{Mode d'alimentation des bovins}

L'alimentation des animaux dans les trois départements a été assurée par le pâturage naturel, les résidus de récolte, le sel et le tourteau de coton (Tableau 3).

Au cours du pâturage, les espèces fourragères les plus appétées dans l'Alibori et dans le Borgou étaient très diversifiées. Par contre, dans l'Atacora, seules trois espèces fourragères ont été utilisées: Andropogon gayanus (100\%), Caya Senegalensis (100\%) et Panicum maximum (25\%). Dans les Départements de l'Alibori et du Borgou, les fourrages les plus appétés étaient: Panicum maximum $\quad(47,7$ vs $76,47 \% ; \quad \mathrm{P}<0,05)$, Andropogon gayanus $\quad(52,5$ vs $100 \%$; $\mathrm{P}<0,05), \quad$ Bracharia sp. $\quad(50$ vs $70,59 \%$; $\mathrm{P}<0,05)$ et Hyparrhenia sp. (42,5 vs 70,59\%; $\mathrm{P}<0,05)$. Dans le Borgou, outre ces espèces, Pennisetum sp. $(70,59 \%)$ et Cajanus cajan (70,59\%) étaient beaucoup consommées au cours du pâturage. Les heures de départ et de retour du pâturage variaient en fonction des régions et des saisons. Pendant la saison des pluies, les animaux étaient conduits au pâturage entre $9 \mathrm{~h}$ et $9 \mathrm{~h} 30 \mathrm{mn}$ et étaient de retour entre $17 \mathrm{~h} 30 \mathrm{mn}$ et $18 \mathrm{~h} 30 \mathrm{mn}$ dans les trois départements. Pendant la saison sèche, les éleveurs conduisaient les animaux au pâturage entre $7 \mathrm{~h}$ et $8 \mathrm{~h}$ et étaient de retour entre $17 \mathrm{~h} 30$ et $19 \mathrm{~h}$.

Les résidus de récolte ont été utilisés comme complément par tous les éleveurs de l'Atacora tandis que dans 1'Alibori, 92,5\% des enquêtés nourrissaient les animaux avec les résidus de récolte contre $81,8 \%$ dans le Borgou. Ces résidus étaient composés de tiges et feuilles de maïs et de sorgho, d'épluchure de manioc, de paille de riz, de graine de coton, de feuille de coton, de fane de niébé et d'épluchure d'igname. Contrairement aux éleveurs de l'Atacora (100\%) et ceux de l'Alibori $(86,84 \%)$ qui utilisaient majoritairement les résidus de récolte du maïs, seule la moitié des éleveurs les utilisaient dans le Borgou (56\%). Les fanes d'arachide étaient utilisées dans les trois départements avec les mêmes fréquences. Les pailles de riz ont été majoritairement $(\mathrm{P}<0,05)$ utilisées pour complémenter les bovins dans l'Alibori $(78,95 \%)$ contrairement à l'Atacora $(33 \%)$ et le Borgou (3,5\%). Les plantes de sorgho ont été plus utilisées $(\mathrm{P}>0,05)$ dans l'Atacora $(95,8 \%)$ et le Borgou $(57,65 \%)$ que dans l'Alibori pour nourrir les animaux. Le tourteau de coton a été uniquement utilisé dans l'Alibori avec une proportion de 7,69\% des éleveurs.

Le stockage d'aliments pour les animaux n'a pas été systématique dans les trois zones d'étude. La quasi-totalité des éleveurs de l'Alibori (95\%) fait le stockage d'aliments, contre $27,06 \%$ dans le Borgou $(\mathrm{P}<0,01)$ et aucun éleveur de bovin du Département de l'Atacora ne le fait. Parmi ceux qui procédaient au stockage d'aliment, les principaux motifs qui soutendent cette pratique étaient la recherche de compléments alimentaires pour les animaux, la prévention de la pénurie de fourrage pendant la saison sèche et l'alimentation des animaux malades. La proportion des éleveurs qui stockaient les 
aliments pour servir de complément alimentaire dans l'Alibori $(42,5 \%)$ a été significativement plus élevée $(\mathrm{P}<0,01)$ que celle des éleveurs du Borgou (22,35\%). Les éleveurs du Département de l'Atacora ne stockaient pas d'aliment à cause de la pratique de la transhumance. Par contre, ceux du Borgou qui ne stockaient pas les aliments ne le faisaient pas par manque de temps $(26 \%)$, par inhabitude $(15 \%)$ et à cause de la transhumance $(25 \%)$.

Les aliments stockés étaient distribués essentiellement pendant la saison sèche quelle que soit la région. Toutefois, la proportion des éleveurs de l'Alibori (95\%) qui le font a été significativement plus élevée $(\mathrm{P}<0,01)$ que celle des éleveurs qui le font dans le Borgou $(27,06 \%)$. Les fréquences de distribution des aliments stockés ont varié en fonction des régions. La plupart des éleveurs du Département de l'Alibori (70\%) servaient l'aliment stocké aux animaux une fois par jour, tandis que seulement $27,4 \%$ des éleveurs $\mathrm{du}$ Borgou le font à cette fréquence $(\mathrm{P}<0,01)$. Toutefois, la proportion des éleveurs qui servaient l'aliment deux fois par jour dans l'Alibori (25\%) a été significativement plus élevée que celles du Borgou et de l'Atacora $(\mathrm{P}<0,05)$. Dans l'Atacora, la distribution des aliments n'a pas été pratiquée par la totalité des éleveurs alors que dans le Borgou, cette proportion a été de $73 \%$.

Les fréquences d'abreuvement des animaux ont varié en fonction des régions $(\mathrm{P}<0,001)$. La moitié des éleveurs du Département de l'Alibori $(50 \%)$ abreuvait les animaux une fois par jour, tandis que la plupart de ceux de l'Atacora $(79,17 \%)$ et du Borgou $(95,29 \%)$, le faisaient deux fois par jour $(\mathrm{P}<0,001)$.Toutefois, la proportion des éleveurs qui servaient l'eau d'abreuvement deux fois par jour dans le Borgou a été significativement plus importante que celle des éleveurs qui le faisait dans l'Atacora $(\mathrm{P}<0,05)$. Par ailleurs, les éleveurs qui servaient l'eau d'abreuvement trois fois par jour ont été plus nombreux dans l'Atacora (20,8\%) que dans l'Alibori $(2,5 \%)$ et n'ont pas été rencontrés dans le Borgou. De manière générale, les animaux s'abreuvaient autour des points d'eau, surtout pendant la saison sèche.

\section{Suivi sanitaire des animaux}

Les cas de maladies ont été signalés par tous les éleveurs enquêtés (tableau 4). Les symptômes rencontrés étaient multiples avec des proportions qui diffèrent en fonction du département. Les cas de diarrhée, de piétin, de toux, de stomatite et d'amaigrissement, ont été plus rencontrés dans le Borgou. Dans l'Atacora, le pica, la toux, la gale et les plaies étaient les pathologies les plus rencontrées. La diarrhée, les amaigrissements, les plaies et la toux représentaient les pathologies dominantes du Département de l'Alibori.

Les traitements contre les signes cliniques sont récapitulés dans le tableau 5.Tous les éleveurs de l'Atacora et du Borgou utilisaient des antibiotiques dans le traitement des pathologies, tandis que dans l'Alibori, $87,18 \%$ en faisaient usage. Les proportions des éleveurs qui traitent les animaux avec les trypanocides ont été plus élevées et ne présentaient pas de différence significative ( $\mathrm{P}>0,05)$ d'un département à l'autre. Les proportions des éleveurs qui déparasitaient leurs animaux dans l'Alibori $(82,05 \%)$ et dans le Borgou $(81,18 \%)$ ne présentaient pas de différence significative. Outre ces divers traitements, dans le Borgou, la vaccination (98,82\%), l'utilisation de cicatrisant (70,59\%), d'insecticide $(11,76 \%)$ et de vitamines ont été pratiqués par les enquêtés. Le traitement des animaux était assuré par la médecine moderne et la pharmacopée. Tous les animaux des trois Départements étaient traités par la médecine moderne. En dehors de ce traitement, 75,29\% des éleveurs du Borgou et 52,50\% de ceux de l'Alibori faisaient appel à la pharmacopée pour le bien être des animaux $(\mathrm{P}<0,05)$. La totalité des éleveurs du Borgou, 91,67\% de ceux de l'Atacora et 77,50\% des enquêtés de l'Alibori, assuraient le traitement des animaux par le vétérinaire ou par eux-mêmes (40\%). Par contre, l'automédication était pratiquée majoritairement dans l'Atacora (95,83\%), cette proportion a été significativement plus élevée que celles du Borgou (76,47\%) et de l'Alibori (37,50\%). 


\section{Aspects économiques}

La vache a coûté plus chère $(\mathrm{P}<0,05)$ dans le Borgou (178785 \pm 14200 F CFA) que dans l'Alibori (122047 $\pm 18800 \mathrm{~F} \mathrm{CFA})$ et l'Atacora (120000 \pm 16750 F CFA). La tendance a été similaire quand on considère le prix d'achat des taureaux. Le taureau a coûté plus cher $(\mathrm{P}<0,05)$ dans le Borgou $(241314,29$ \pm 20250 F CFA) que dans l'Alibori (155590 \pm $25541 \mathrm{~F} \mathrm{CFA})$ et l'Atacora $(180000 \pm 29800$ F CFA).

A la vente, le taureau coûtait plus cher $(\mathrm{P}<0,01)$ dans le Borgou (285798 $\pm 14145 \mathrm{~F}$ CFA) que dans l'Atacora (201667 $19795 \mathrm{~F}$ CFA) alors que dans l'Alibori, ce prix a été intermédiaire entre ceux du Borgou et de l'Atacora et n'a pas été significativement différent (242500 \pm 16163 F CFA). De même, les prix de vente des vaches ont été plus élevés $(\mathrm{P}<0,001)$ dans le Borgou (197955 \pm 10490 F CFA) que dans l'Atacora (125416 \pm 14350 F CFA) et dans l'Alibori (150333 \pm $11700 \mathrm{~F} \mathrm{CFA})$. Le prix de vente moyen des veaux a été de $68000 \pm 5700 \mathrm{~F}$ CFA et celui des velles, $82222 \pm 5800$ F CFA dans l'Alibori. Les veaux et les velles coûtaient respectivement $58333 \pm 3700 \mathrm{~F} \mathrm{CFA}$ et 81458 \pm 3500 F CFA dans l'Atacora. Les prix de vente des veaux et des velles ont été relativement plus bas $(\mathrm{P}<0,001)$ dans le Borgou que dans les autres départements avec des prix de $39390 \pm 2814$ F CFA et $49939 \pm 2700$ F CFA, respectivement pour les veaux et les velles.

La quantité moyenne journalière de lait produit par éleveur diffère significativement en fonction des départements $(\mathrm{P}<0,05)$. Dans l'Atacora, la production moyenne journalière de lait par éleveur (19,75 litres) a été plus importante $(\mathrm{P}<0,05)$ que celles enregistrées dans l'Alibori (12,81 litres) et le Borgou (14,28 litres). Par contre, le litre de lait a été plus cher dans le Borgou (298 F CFA) que dans l'Alibori (225 F CFA) et dans l'Atacora (250 F CFA).

\section{Typologie des élevages}

Trois axes ont été retenus pour l'interprétation des résultats de l'analyse factorielle des correspondances $\left(\chi^{2}=1199,8\right)$.
Le premier axe factoriel concernait les éleveurs de Péréré, de Banikoara et un groupe d'éleveurs de Tchaourou. Le second axe discriminait les éleveurs de Kèrou dont l'un des objectifs de production a été de fournir des bœufs de trait aux agriculteurs. Enfin, les éleveurs d'embouche de Parakou étaient très bien représentés sur le troisième axe factoriel. Une classification ascendante hiérarchique réalisée sur les coordonnées de ces axes a permis de regrouper les élevages en trois catégories. La Figure 2 présente la typologie des élevages sur le plan factoriel représenté par les axes 1 et 3 . Trois types d'élevages ont été identifiés :

Les élevages de type 1 ont été rencontrés dans les Communes de Pèrèrè, de Banikoara et chez un groupe d'éleveurs de Tchaourou (Tchaourou 1). Ils étaient des éleveurs professionnels ou des agriculteurs et font le plus souvent un élevage de prestige. Le commerce a été une des activités secondaires. L'objectif de production était le lait. Ils vendaient très peu d'animaux et ceci se faisait seulement en cas de besoin ponctuel d'argent. Le traitement des animaux malades se faisait par le vétérinaire. Le suivi des animaux se faisait par des employés à plein temps et le propriétaire s'en occupait à mi-temps. Ils étaient le plus souvent des Peul ou des Gando.

Les éleveurs de bovins de Kèrou et du second groupe d'éleveurs de Tchaourou (Tchaourou 2) étaient des éleveurs professionnels et secondairement des agriculteurs. Ils vendaient des animaux pendant les périodes de fête sur la base de leur embonpoint. Les propriétaires d'animaux se chargaient du suivi à plein temps, leurs épouses, à mi-temps à Kèrou et à plein temps à Tchaourou 2. Les éleveurs de Kèrou et de Tchaourou 2 étaient pour la plupart des Peulh. Ils vendaient des bovins de boucherie, du lait et des animaux de trait. Ils produisaient du fromage et consommaient le lait provenant de leur exploitation. Dans leurs troupeaux, les jeunes taurillons élevés en embouche herbagère sur pâturage naturel avec parfois une distribution de complément alimentaire ou de résidus de récolte ont été fréquemment rencontrés. Ils vendaient les animaux à des 
moments propices pour avoir le maximum d'argent. Ils vendaient également les animaux malades. Ce type d'élevage est qualifié de type 2 .

Le type 3 concerne les éleveurs de bovins de Parakou qui ont le commerce comme activité principale et secondairement, ils étaient des éleveurs ou bouchers. Ils élevaient des animaux d'embouche et par conséquent, ne produisaient pas du lait, n'en vendaient pas et ne le transformaient pas. Leur but était de produire de la viande à travers l'engraissement des animaux. Ils étaient Bariba ou Dendi.

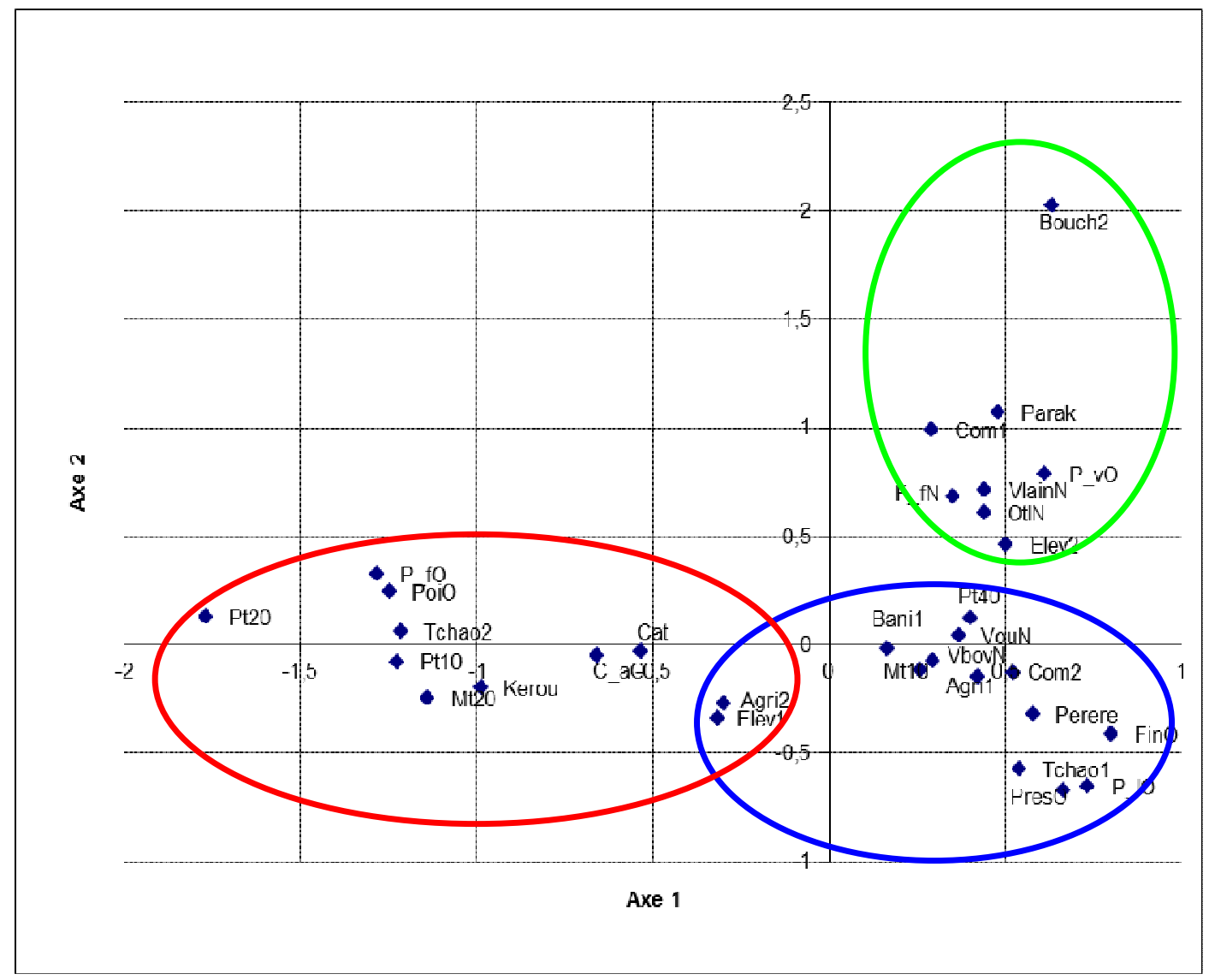

Les Commune d'enquête sont : Banikoara scindé en deux groupes d'éleveurs (Bani1 et Bani2), Tchaourou scindé en deux groupes d'éleveurs (Tchao1 et Tchao2), Pèrèrè et Kèrou. L'activité principale des éleveurs est l'élevage (Elev1), l'agriculture (Agri1) ou le commerce (Com1). L'activité secondaire est l'élevage (Elev2), agriculture (agri2) ou le commerce (Com2). Les bovins sont vendus (VbovO), le lait est vendu (VlaiO), les mâles sont utilisés dans la culture attelée $(\mathrm{CaO})$, le lait est utilisé dans la fabrication du fromage (FfO), le lait est auto-consommé (OtlO). La vente se fait selon l'âge (AgeO), en période de fête ( $\mathrm{PfO}$ ), en fonction du poids ( $\mathrm{PoiO}$ ), en cas de pathologies (PatO) ou pour des besoins financiers (FinO). L'objectif de production est le lait $(\mathrm{PlO})$, la viande $(\mathrm{PvO})$, le lait et la viande $(\mathrm{Plv})$, la culture attelée $(\mathrm{CatO})$ ou le prestige $(\mathrm{PresO})$. Pt1O : le propriétaire s'occupe des animaux à plein temps; $\mathrm{Mt} 1 \mathrm{O}$ : le propriétaire s'occupe des animaux à mi-temps, Pt2O : l'épouse du propriétaire s'occupe des animaux à plein temps; Mt2O : l'épouse du propriétaire s'occupe des animaux à mitemps; $\mathrm{Pt} 4 \mathrm{O}$ : les employés s'occupent des animaux à plein temps; Mt4O : les employés s'occupent des animaux à mitemps ; VetO : le mode de traitement est moderne ; CopO : la pharmacopée est le mode de traitement ; VO : le traitement des animaux est assuré par le vétérinaire ; VouO : le traitement des animaux est assuré par l'éleveur.

Figure 2: Typologie des élevages de bovin de race Borgou sur les axes factoriels 1 et 2. 
Tableau 1: Structure des troupeaux de bovins dans les Départements du Borgou, de l'Atacora et de l'Alibori.

\begin{tabular}{|c|c|c|c|c|}
\hline Variables & $\begin{array}{r}\text { Alibori } \\
\text { Moyenne } \pm \text { ES }\end{array}$ & $\begin{array}{r}\text { Atacora } \\
\text { Moyenne } \pm \text { ES }\end{array}$ & $\begin{array}{c}\text { Borgou } \\
\text { Moyenne } \pm \text { ES }\end{array}$ & $\begin{array}{c}\text { Test de } \\
\text { Significativité }\end{array}$ \\
\hline Taureau (3 ans et plus) & $3,83 \pm 0,86 a$ & $2,42 \pm 1,11 \mathrm{a}$ & $4,13 \pm 0,59 a$ & NS \\
\hline Vache non gestante & $5,18 \pm 1,11 \mathrm{~b}$ & $12,7 \pm 1,44 \mathrm{a}$ & $7,71 \pm 0,76 b$ & $* * *$ \\
\hline Vache gestante & $9,88 \pm 1,55 b$ & $18,5 \pm 2,01 \mathrm{a}$ & $9,98 \pm 1,07 b$ & $* * *$ \\
\hline Vache allaitante & $13,7 \pm 1,87 \mathrm{a}$ & $18,8 \pm 2,42 \mathrm{a}$ & $16,3 \pm 1,28 \mathrm{a}$ & NS \\
\hline Bouvillon 1 (1 à 2 ans) & $0,03 \pm 0,07 \mathrm{a}$ & $0,21 \pm 0,1 \mathrm{a}$ & $0,08 \pm 0,05 a$ & NS \\
\hline Bouvillon 2 (2 à 3 ans) & $0,05 \pm 0,13 \mathrm{a}$ & $0 \pm 0,17 \mathrm{a}$ & $0,28 \pm 0,09 \mathrm{a}$ & NS \\
\hline Vache & $29,3 \pm 3,91 \mathrm{a}$ & $37,17 \pm \mathrm{a}$ & $32,8 \pm 2,65 \mathrm{a}$ & NS \\
\hline Veau/Velle (0 à 1 an) & $12,4 \pm 1,92 b$ & $18,8 \pm 2,42 \mathrm{a}$ & $16,3 \pm 1,28 \mathrm{ab}$ & NS \\
\hline Genisse $1 /$ Taurillon 1 ( 1 à 2 ans) & $7,43 \pm 1,75 b$ & $14 \pm 2,18 \mathrm{a}$ & $10,7 \pm 1,16 \mathrm{ab}$ & NS \\
\hline Genisse 2/Taurillon 2 (2 à 3 ans) & $7,43 \pm 2 \mathrm{a}$ & $10,3 \pm 2,41 \mathrm{a}$ & $9,7 \pm 1,28 \mathrm{a}$ & NS \\
\hline Bœufs (plus de 3 ans) & $0,45 \pm 0,19 \mathrm{a}$ & $0,0 \pm 0,24 \mathrm{a}$ & $0,44 \pm 0,13 \mathrm{a}$ & NS \\
\hline
\end{tabular}


Tableau 2: Objectifs de production des éleveurs de bovins de race Borgou par département.

\begin{tabular}{|c|c|c|c|c|c|}
\hline \multicolumn{2}{|l|}{ Variables } & $\begin{array}{c}\text { Alibori } \\
\text { Fréquence } \pm \text { IC }(\%)\end{array}$ & $\begin{array}{c}\text { Atacora } \\
\text { Fréquence } \pm \text { IC }(\%)\end{array}$ & $\begin{array}{c}\text { Borgou } \\
\text { Fréquence } \pm \text { IC }(\%)\end{array}$ & $\begin{array}{c}\text { Test de } \\
\text { Significativité }\end{array}$ \\
\hline \multirow{5}{*}{ But de la Création } & & & & & \\
\hline & Production de lait & $37,5 \pm 15 b$ & $0 \pm 0,00 \mathrm{c}$ & $61,2 \pm 10,4 \mathrm{a}$ & $* * *$ \\
\hline & Production de viande & $12,5 \pm 10,3 \mathrm{ab}$ & $0 \pm 0,00 \mathrm{~b}$ & $25,9 \pm 9,31 \mathrm{a}$ & $* *$ \\
\hline & Culture attelée & $92,5 \pm 8,16 \mathrm{a}$ & $95,8 \pm 8 \mathrm{a}$ & $1,18 \pm 2,3 b$ & $* * *$ \\
\hline & Prestige & $40 \pm 15,2 \mathrm{a}$ & $0 \pm 0,00 \mathrm{~b}$ & $48,3 \pm 10,6 \mathrm{a}$ & $* * *$ \\
\hline \multirow{4}{*}{$\begin{array}{l}\text { Utilisation des } \\
\text { animaux et de leurs } \\
\text { produits }\end{array}$} & Vente des bovins & $72,5 \pm 13,8 b$ & $100 \pm 0,00 \mathrm{a}$ & $98,8 \pm 2,3 \mathrm{a}$ & $* * *$ \\
\hline & Culture attelée & $77,5 \pm 13 b$ & $100 \pm 0,00 \mathrm{a}$ & $3,53 \pm 3,92 \mathrm{c}$ & $* * *$ \\
\hline & Fabrication du fromage & $37,5 \pm 15 c$ & $95,8 \pm 8 \mathrm{a}$ & $75,3 \pm 9,17 \mathrm{~b}$ & $* * *$ \\
\hline & Auto-consommation du lait & $65 \pm 14,8 b$ & $100 \pm 0,00 \mathrm{a}$ & $75,3 \pm 9,17 \mathrm{~b}$ & $* *$ \\
\hline \multirow{5}{*}{$\begin{array}{l}\text { Critères de vente } \\
\text { des animaux }\end{array}$} & Age & $80,6 \pm 12,3 b$ & $100 \pm 0,00 \mathrm{a}$ & $41,2 \pm 10,5 \mathrm{c}$ & $* * *$ \\
\hline & Période de fête & $5,56 \pm 5,56 \mathrm{~b}$ & $0 \pm 0,00 \mathrm{~b}$ & $29,4 \pm 9,69 a$ & $* * *$ \\
\hline & Poids & $0 \pm 0,00 \mathrm{c}$ & $100 \pm 0,00 \mathrm{a}$ & $32,9 \pm 9,99 b$ & $* * *$ \\
\hline & Pathologies & $0 \pm 0,00 \mathrm{~b}$ & $41,7 \pm 19,7 \mathrm{a}$ & $30,6 \pm 9,8 \mathrm{a}$ & $* * *$ \\
\hline & Culture attelée & $2,86 \pm 5,17 \mathrm{a}$ & $0 \pm 0,00 \mathrm{a}$ & $0 \pm 0,00 \mathrm{a}$ & NS \\
\hline \multirow{6}{*}{$\begin{array}{l}\text { Cause de vente des } \\
\text { animaux }\end{array}$} & Argent & $80 \pm 12,4 \mathrm{~b}$ & $58,3 \pm 19,7 \mathrm{~b}$ & $95,2 \pm 4,53 a$ & \multirow{6}{*}{$* * *$} \\
\hline & Déstockage & $2,86 \pm 5,17 \mathrm{a}$ & $0 \pm 0,00 \mathrm{a}$ & $0 \pm 0,00 \mathrm{a}$ & \\
\hline & Maladie & $0 \pm 0,00 \mathrm{a}$ & $0 \pm 0,00 \mathrm{a}$ & $1,19 \pm 2,31 \mathrm{a}$ & \\
\hline & Renouvelle cheptel & $0 \pm 0,00 \mathrm{a}$ & $0 \pm 0,00 \mathrm{a}$ & $3,57 \pm 3,94 \mathrm{a}$ & \\
\hline & Vieux animaux et besoin d'argent & $5,71 \pm 7,19 b$ & $41,7 \pm 19,7 \mathrm{a}$ & $0 \pm 0,00 \mathrm{c}$ & \\
\hline & Vieux animaux & $11,4 \pm 9,86 a$ & $0 \pm 0,00 \mathrm{~b}$ & $0 \pm 0,00 \mathrm{~b}$ & \\
\hline
\end{tabular}


I. YOUSSAO ABDOU KARIM et al. / Int. J. Biol. Chem. Sci. 7(1): 125-146, 2013

Tableau 3: Mode d'alimentation des bovins de race Borgou.

\begin{tabular}{|c|c|c|c|c|c|}
\hline \multirow[t]{2}{*}{ Variable } & & $\begin{array}{l}\text { Alibori } \\
\end{array}$ & Atacora & Borgou & Test de significativité \\
\hline & & Fréquence \pm IC (\%) & Fréquence $\pm \mathrm{IC}(\%)$ & Fréquence \pm IC (\%) & \\
\hline \multirow{4}{*}{$\begin{array}{l}\text { Aliments donnés } \\
\text { aux animaux }\end{array}$} & Fourrage & $100 \pm 0,00 \mathrm{a}$ & $100 \pm 0,00 \mathrm{a}$ & $100 \pm 0,00 \mathrm{a}$ & - \\
\hline & Résidus de récolte & $92,5 \pm 8,16 \mathrm{ab}$ & $100 \pm 0,00 \mathrm{a}$ & $81,2 \pm 8,31 \mathrm{~b}$ & - \\
\hline & Tourteau de coton & $7,69 \pm 8,26 \mathrm{a}$ & $0 \pm 0,00 \mathrm{a}$ & $0 \pm 0,00 \mathrm{~b}$ & - \\
\hline & Sel & $100 \pm 0,00 \mathrm{a}$ & $100 \pm 0,00 \mathrm{a}$ & $100 \pm 0,00 \mathrm{a}$ & - \\
\hline \multirow{10}{*}{ Types de fourrage } & Panicum maximum & $47,5 \pm 15,48 b$ & $25 \pm 17,3 b$ & $76,5 \pm 9,02 \mathrm{a}$ & - \\
\hline & Andropogon gayanus & $52,5 \pm 15,48 b$ & $100 \pm 0,00 \mathrm{a}$ & $100 \pm 0,00 \mathrm{a}$ & - \\
\hline & Bracharia sp & $50 \pm 15,5 b$ & $0 \pm 0,00 \mathrm{c}$ & $70,6 \pm 9,69 \mathrm{a}$ & - \\
\hline & Caya senegalensis & $2,50 \pm 4,48 \mathrm{c}$ & $100 \pm 0,00 \mathrm{a}$ & $27,7 \pm 9,44 b$ & - \\
\hline & Sida acuta & $2,50 \pm 4,84 \mathrm{a}$ & $0 \pm 0,00 \mathrm{a}$ & $0 \pm 0,00 \mathrm{a}$ & - \\
\hline & Pennisetum sp & $35 \pm 14,78 b$ & $0 \pm 0,00 \mathrm{c}$ & $70,6 \pm 9,69 a$ & - \\
\hline & Hyparrhenia sp & $42,5 \pm 15,3 b$ & $0 \pm 0,00 \mathrm{c}$ & $70,6 \pm 9,69 a$ & - \\
\hline & Setaria $\mathrm{sp}$ & $7,50 \pm 8,16 \mathrm{a}$ & $0 \pm 0,00 \mathrm{a}$ & $0 \pm 0,00 \mathrm{~b}$ & - \\
\hline & Leucena leucocephala & $2,50 \pm 8,84 \mathrm{a}$ & $0 \pm 0,00 \mathrm{a}$ & $0 \pm 0,00 \mathrm{a}$ & - \\
\hline & Cajanus cajan & $2,50 \pm 4,84 \mathrm{~b}$ & $0 \pm 0,00 \mathrm{~b}$ & $70,6 \pm 9,69 \mathrm{a}$ & - \\
\hline \multirow{12}{*}{ Résidus de récolte } & Plante de maïs & $86,8 \pm 10,5$ a & $100 \pm 0,00 \mathrm{a}$ & $56,5 \pm 10,5 b$ & $* * *$ \\
\hline & Plante de sorgho & $15,8 \pm 11,3 b$ & $95,8 \pm 8 \mathrm{a}$ & $57,6 \pm 10,5 \mathrm{a}$ & $* * *$ \\
\hline & Fane d'arachide & $73,7 \pm 16,6 \mathrm{a}$ & $54,2 \pm 19,9 \mathrm{a}$ & $69,4 \pm 9,8 \mathrm{a}$ & NS \\
\hline & Epluchure de manioc & $0 \pm 0,00 \mathrm{~b}$ & $4,17 \pm 8 b$ & $48,2 \pm 10,62 \mathrm{a}$ & $* * *$ \\
\hline & Paille de riz & $78,9 \pm 12,6 \mathrm{a}$ & $33,3 \pm 18,9 b$ & $3,53 \pm 3,92 \mathrm{c}$ & $* * *$ \\
\hline & Graine de coton & $2,56 \pm 4,9 b$ & $45,8 \pm 19,9 \mathrm{a}$ & $2,35 \pm 3,22 b$ & $* * *$ \\
\hline & Feuille de soja & $0 \pm 0,00 \mathrm{a}$ & $0 \pm 0,00 \mathrm{a}$ & $1,18 \pm 2,30 \mathrm{a}$ & NS \\
\hline & Feuille de coton & $5,13 \pm 6,84 b$ & $100 \pm 0,00 \mathrm{a}$ & $0 \pm 0,00 \mathrm{c}$ & $* * *$ \\
\hline & Fane de niébé & $31,6 \pm 14,41 \mathrm{a}$ & $0 \pm 0,00 \mathrm{~b}$ & $50,6 \pm 10,6 \mathrm{a}$ & $* * *$ \\
\hline & Drèche & $0 \pm 0,00 \mathrm{a}$ & $0 \pm 0,00 \mathrm{a}$ & $1,18 \pm 2,30 \mathrm{a}$ & NS \\
\hline & Epluchure d'igname & $0 \pm 0,00 \mathrm{~b}$ & $0 \pm 0,00 \mathrm{~b}$ & $45,9 \pm 10,6 \mathrm{a}$ & $* * *$ \\
\hline & Haricot & $15,8 \pm 11,3 \mathrm{a}$ & $8,33 \pm 11,1 \mathrm{a}$ & $0 \pm 0,00 \mathrm{~b}$ & $* *$ \\
\hline
\end{tabular}


I. YOUSSAO ABDOU KARIM et al. / Int. J. Biol. Chem. Sci. 7(1): 125-146, 2013

Tableau 4: Pathologies et signes cliniques rencontrés dans les élevages de bovins.

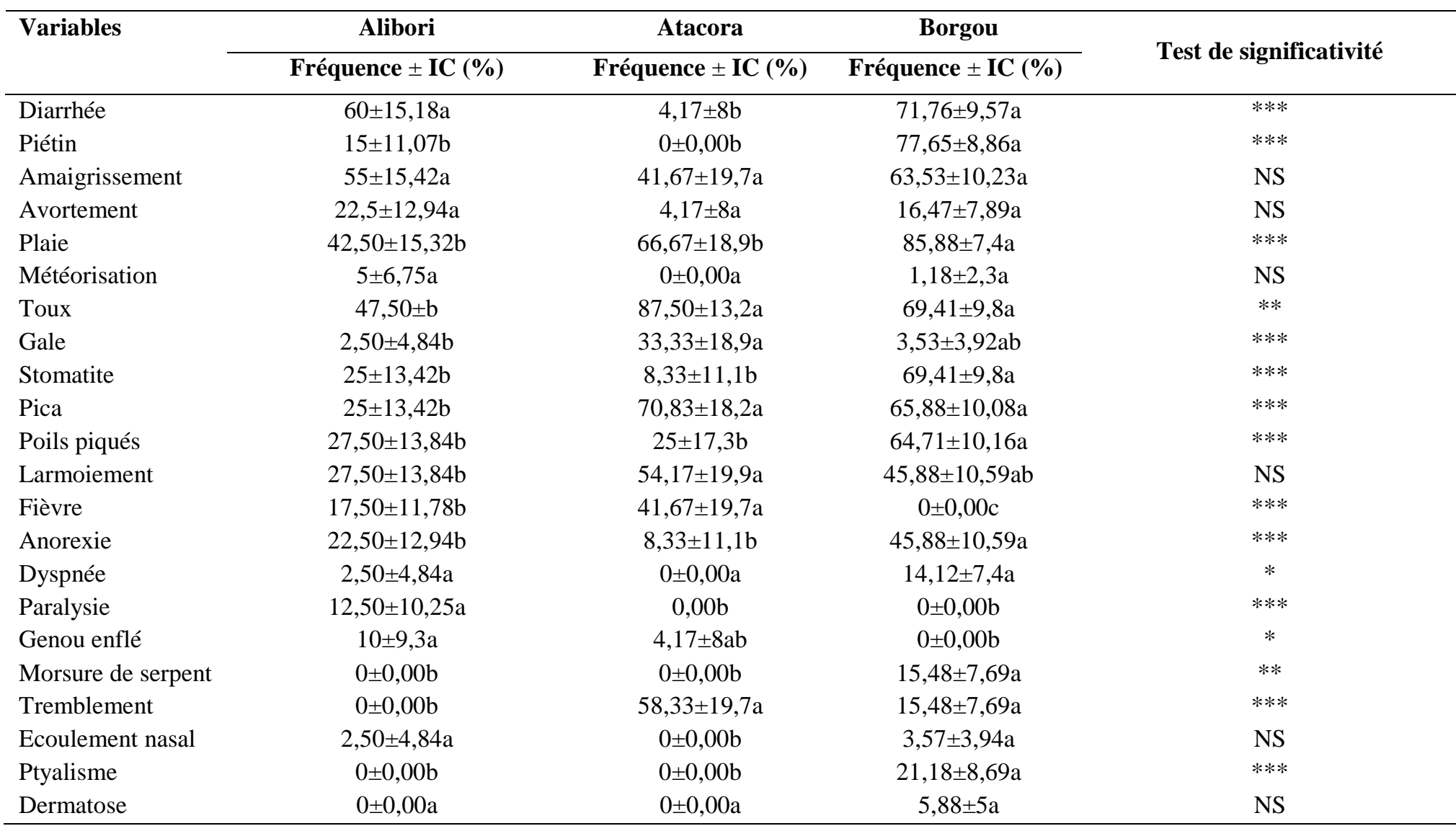

IC : Intervalle de confiance $; \mathrm{NS}: \mathrm{P}>0,05 ; *: \mathrm{P}<0,05 ; * *: \mathrm{P}<0,01 ; * * *: \mathrm{P}<0,001 ;$ les pourcentages de la même ligne suivis de la même lettre ne diffèrent pas significativement au seuil de $5 \%$. 
Tableau 5: Traitements contre les signes cliniques.

\begin{tabular}{|c|c|c|c|c|c|}
\hline \multirow[t]{2}{*}{ Variables } & & Alibori & Atacora & Borgou & \multirow{2}{*}{$\begin{array}{c}\text { Test de } \\
\text { significativité }\end{array}$} \\
\hline & & Fréquence \pm IC (\%) & Fréquence \pm IC (\%) & Fréquence \pm IC (\%) & \\
\hline \multirow{8}{*}{$\begin{array}{l}\text { Produits } \\
\text { vétérinaires utilisés }\end{array}$} & Antibiothérapie & $87,2 \pm 10,4 b$ & $100 \pm 0,00 \mathrm{a}$ & $100 \pm 0,00 \mathrm{a}$ & $* * *$ \\
\hline & Vaccination & $0 \pm 0,00 \mathrm{~b}$ & $0 \pm 0,00 \mathrm{~b}$ & $98,8 \pm 2,3 \mathrm{a}$ & $* * *$ \\
\hline & Trypanocide & $97,4 \pm 4,89 b$ & $100 \pm 0,00 \mathrm{a}$ & $98,8 \pm 2,3 \mathrm{a}$ & NS \\
\hline & Vitamine & $2,56 \pm 4,89 \mathrm{a}$ & $0 \pm 0,00 \mathrm{a}$ & $12,9 \pm 7,14 \mathrm{a}$ & $*$ \\
\hline & Anti diarrhéique & $0 \pm 0,00 \mathrm{~b}$ & $0 \pm 0,00 \mathrm{~b}$ & $3,53 \pm 3,92 \mathrm{a}$ & NS \\
\hline & Déparasitage & $82,1 \pm 11,9 \mathrm{a}$ & $0 \pm 0,00 \mathrm{~b}$ & $81,2 \pm 8,31 \mathrm{a}$ & $* * *$ \\
\hline & Insecticide & $0 \pm 0,00 \mathrm{~b}$ & $0 \pm 0,00 \mathrm{a}$ & $11,8 \pm 6,85 \mathrm{a}$ & $*$ \\
\hline & Cicatrisant & $0 \pm 0,00 \mathrm{~b}$ & $0 \pm 0,00 \mathrm{~b}$ & $70,6 \pm 9,69 a$ & $* * *$ \\
\hline \multirow{2}{*}{$\begin{array}{l}\text { Mode de } \\
\text { traitement }\end{array}$} & Vétérinaire & $100 \pm 0,00 \mathrm{a}$ & $100 \pm 0,00 \mathrm{a}$ & $100 \pm 0,00 \mathrm{a}$ & - \\
\hline & Pharmacopée & $52,5 \pm 15,5 b$ & $0 \pm 0,00 \mathrm{c}$ & $75,3 \pm 9,17 \mathrm{a}$ & $* * *$ \\
\hline \multirow{2}{*}{$\begin{array}{l}\text { Traitement } \\
\text { des animaux }\end{array}$} & Vétérinaire & $77,5 \pm 12,9 b$ & $91,67 \pm 11,1 b$ & $100 \pm 0,00 \mathrm{a}$ & $* * *$ \\
\hline & Eleveur & $37,5 \pm 15 c$ & $95,8 \pm 8 \mathrm{a}$ & $76,5 \pm 9,02 b$ & $* * *$ \\
\hline
\end{tabular}

IC : Intervalle de confiance $; \mathrm{NS}: \mathrm{P}>0,05 ; *: \mathrm{P}<0,05 ; * *: \mathrm{P}<0,01 ; * * *: \mathrm{P}<0,001 ;$ les pourcentages de la même ligne suivis de la même lettre ne diffèrent pas significativement au seuil de $5 \%$ 


\section{DISCUSSION}

Statut de l'éleveur

A l'issue de notre étude, il ressort que l'élevage des bovins de race Borgou était majoritairement pratiqué par les Peulh. Les éleveurs rencontrés au cours de notre étude pratiquaient pour la plupart la religion musulmane et étaient non scolarisés. Ces résultats sont similaires à ceux obtenus par Alkoiret et al. (2009) lors de l'étude sur la typologie des élevages de bovins de la Commune de Gogounou. Le profil des éleveurs de bovins des Départements de l'Alibori, de l'Atacora et du Borgou est similaire à celui des aviculteurs traditionnels des mêmes zones (Houessionnon, 2011). Les travaux réalisés par Somda et al. (2004) en Guinée-Bissau ont aussi confirmé l'importance des Peulh dans l'élevage des bovins avec une proportion de 96,2\% des éleveurs.

Les femmes éleveurs de bovins ne se retrouvaient que dans le Borgou et étaient très minoritaires. La faiblesse de cette proportion est due à des paramètres sociologiques. L'organisation sociale des Peulh attribue l'élevage des bovins exclusivement aux hommes (Bierschenk et Forster, 2004). De plus, le système d'élevage traditionnel des bovins est caractérisé par la pratique de la transhumance, activité qui reste l'apanage des hommes (Dehoux et Hounsou-Vê, 1993). La même observation a été faite en GuinéeBissau au sein des élevages bovins laitiers (Somda et al., 2004).

\section{Activités des éleveurs}

Les activités pratiquées par les éleveurs dans la zone d'étude sont de différents types. L'élevage a été l'activité principale dominante dans les trois Départements. La seconde activité principale la plus pratiquée dans l'Alibori et dans le Borgou a été l'agriculture. L'activité secondaire dominante dans les trois zones d'étude est l'agriculture avec une fréquence plus élevée dans l'Atacora. L'association entre l'élevage et l'agriculture qui a été mise en évidence par cette étude est similaire au constat fait par Alkoiret et al.
(2009). Le commerce, la fonction publique, l'artisanat et la boucherie sont également des activités menées par les éleveurs du septentrion. Cette diversité des activités principales des éleveurs de bovins témoigne que l'élevage des bovins est rarement le seul moyen de subsistance du ménage des éleveurs. En effet, l'élevage représente l'une des nombreuses activités intégrées et complémentaires du système fermier qui contribue à son bien-être général (FAO, 2004). Dans ce contexte, l'élevage des bovins au Bénin permet la diversification des revenus des populations rurales.

\section{Constitution du troupeau}

Le mode d'acquisition des animaux est très diversifié. La plupart des éleveurs de l'Alibori et de l'Atacora ont acquis leurs premiers animaux par don; alors que dans le Borgou, l'acquisition des premiers animaux se fait par achat au comptant qui représente le premier mode de constitution du cheptel dans cette région. Ces résultats sont comparables à ceux rapportés par Houessionnon (2011) sur les systèmes d'élevage de volailles au Bénin et par Alkoiret et al. (2009) sur les systèmes d'élevage de bovin dans la Commune de Gogounou.

Le financement des fermes d'élevage est assuré par trois sources: la famille, l'éleveur et rarement les appuis extérieurs, confirmant ainsi que le système d'élevage adopté en milieu paysan est donc de type traditionnel et surtout familial (Koutinhouin et al., 2010), et révèle que les organismes nationaux et internationaux de financement et d'appui à l'élevage dans le septentrion sont insuffisants et peu efficients.

\section{Structure du troupeau}

Les troupeaux rencontrés dans la zone d'étude sont de type extensif et traditionnel. Ce qui confirme les résultats de Koutinhouin et al. (2010) qui rapportent que le système d'élevage adopté en milieu paysan est de type extensif et traditionnel. L'effectif moyen de bovins dans les élevages enquêtés a été de 57 bovins dans l'Alibori, 83 bovins dans 
l'Atacora et 63 bovins dans le Borgou. Dans la Commune de Gogounou située dans le Département de l'Alibori, l'effectif moyen de bovins dans les élevages enquêtés est de $78 \pm$ 56 têtes (Alkoiret et al., 2009). La taille moyenne des troupeaux dans la Commune de Gogounou est supérieure à celles des Départements de l'Alibori et du Borgou.

\section{Caractéristiques des habitats}

Quant aux caractéristiques des habitats, elles sont de type traditionnel dans les trois régions. A la Ferme d'Elevage de l'Okpara où l'élevage est de type semi amélioré, le parc de nuit est construit en bois ou en béton entouré par des fils de fer barbelés (Youssao et al., 2009). Ces parcs sont plus confortables que ceux des élevages traditionnels.

\section{Objectifs de production}

Les mobiles qui motivent la création d'une ferme d'élevage de bovins sont diversifiés. Dans l'Alibori, les éleveurs de bovins font leur élevage pour pratiquer la culture attelée, pour produire du lait et pour le prestige. Ces éleveurs ne sont pas disposés à se séparer de leurs animaux, ce qui explique la faible proportion d'éleveurs qui font la production de viande. Par contre, dans le Borgou, la majorité des éleveurs font une production mixte (viande et lait). Par ailleurs, aucun éleveur de l'Atacora n'élève les animaux pour le prestige, ni pour la production $\mathrm{du}$ lait uniquement ou la production de viande uniquement, mais ils font tous la production de bovins à la fois pour le lait et la viande. Le mode d'exploitation basé sur le prestige est également observé au Madagascar où certains éleveurs attribuent une valeur plus culturelle que financière à leurs troupeaux (Solomalala et al., 2010).

Quant à l'utilisation des animaux et de leurs productions, tous les éleveurs de bovins produisent principalement pour la vente et secondairement pour la culture attelée et l'autoconsommation. Ces objectifs de production et ces modes d'utilisation des animaux et de leurs productions montrent que ces trois départements du Nord-Bénin sont essentiellement agricoles. A Madagascar, la production de lait contribue pour 45 à $53 \%$ des revenus des éleveurs de bovins de Menabe central (Solomalala et al., 2010).

Les critères qui motivent la sortie des animaux sont sensiblement les mêmes dans les trois Départements et sont: l'âge, les problèmes financiers et les cas de morbidité chez les bovins. La cause fondamentale de vente des animaux dans les trois Départements est la recherche de l'argent. Le déstockage, les pathologies et le renouvellement du cheptel constituent les causes secondaires de la vente des animaux. Ces résultats sont comparables à ceux enregistrés par Houessionnon (2011) sur la volaille dans les mêmes zones agroécologiques.

\section{Mode d'alimentation des bovins}

L'alimentation des animaux est assurée dans les trois régions par le fourrage en pâturage naturel, des résidus de récolte et le sel de cuisine. Les mêmes constats ont été faits par Alkoiret et al. (2009) lors de leurs travaux réalisés dans la commune de Gogounou. Les fourrages utilisés dans l'Alibori et le Borgou sont très divers et constitués par ordre d'importance de: Andropogon gayanus, Panicum maximum, Hyparenia spp, Penisetum spp, Setaria spp, Leucena spp, tiges de coton, etc. Par contre, dans l'Atacora, seules trois espèces fourragères sont utilisées: Andropogon gayanus, Caya senegalensis et Panicum maximum. Les études réalisées par Alkoiret et Ggangboche (2005) et Bierschenk et Forster (2004) sur l'organisation sociale des Peulh, ont confirmé l'importance des plantes fourragères dans l'alimentation des bovins. Les plantes dont ils ont fait cas sont les mêmes que celles rencontrées dans le cadre de cette étude. L'alimentation sur pâturage naturel a été observée par Koutinhouin et al. (2010) qui rapportent que pendant la saison des pluies, les animaux sont conduits au pâturage par de jeunes bouviers et que pendant la saison sèche, le seul mode de 
conduite des animaux demeure la vaine pâture.

Divers résidus de récolte étaient utilisés dans l'alimentation des bovins. Il s'agit notamment de maïs, de sorgho, des fanes d'arachides, des pailles de riz, des épluchures de manioc, des fanes de coton, de niébé et d'haricot, puis des graines de coton. Ces observations sont similaires à celles réalisées par Koutinhouin et al. (2010) sur l'alimentation des bovins de race Borgou en élevage traditionnel et Bani Kodji (2011) sur la gestion de l'alimentation des bovins de race Borgou dans les Communes de Sinendé dans le Borgou et de Ouassa-Péhunco dans l'Atacora. Une fois les récoltes terminées, l'alimentation des animaux est surtout constituée des résidus de récoltes comme les tiges de maïs, de mil, de sorgho et du coton (Yacoubou, 2011). Les animaux se déplacent sur de longues distances afin de glaner quelques repousses de graminées ou longent les vallées des cours d'eau pour consommer les espèces fourragères qui les jalonnent. Il faut cependant noter que la pratique de l'émondage des arbres fourragers vient combler en partie le déficit fourrager (Bani Kodji, 2011).

La totalité des éleveurs enquêtés se sert de l'eau pour l'abreuvement des animaux. Les fréquences de distribution de l'eau varient en fonction des régions. Cette différence s'explique par le fait que l'abreuvement des animaux se fait à partir des cours d'eaux. Or, la répartition des points d'eau naturelle n'est pas identique dans la zone d'étude. Le même constat a été fait par Adjou Moumouni (2006) lors de son étude sur l'évaluation des performances zootechniques des bovins de race Borgou en sélection à la ferme d'élevage de l'Okpara au Bénin.

Les heures de départ au pâturage et de retour du pâturage varient en fonction des régions et des saisons dans la présente étude. Selon Koutinhouin et al. (2010), après la traite matinale, les animaux sont conduits au pâturage vers 8-9 heures par de jeunes bouviers, sous la responsabilité d'un bouvier professionnel, et reviennent vers 18-19 heures.
Pendant la saison sèche où les parcours naturels restent nus après le passage des feux de brousse annuels, le seul mode de conduite des animaux demeure la vaine pâture comme l'a aussi remarqué Bani Kodji (2011). D'après Dehoux et Hounsou-Vê (1993), en moyenne, les bovins sortent 8 heures par jour dans le Borgou. Toutefois, pendant la saison des pluies, ce temps est ramené à 6 heures pour cause de travaux champêtres et de l'abondance du pâturage naturel.

Le stockage d'aliments pour les animaux n'est pas systématique dans la zone d'étude. Certains des éleveurs enquêtés pratiquent l'élevage extensif, pour lequel l'alimentation est surtout basée sur le pâturage naturel. L'étude réalisée par Alkoiret et al. (2009) a fait les mêmes observations. Les quasi-totalités des éleveurs de l'Alibori font le stockage d'aliments contre le tiers dans le Borgou. Les aliments stockés sont distribués essentiellement pendant la saison sèche quelque soit la région. Ce qui confirme le lien fait par Adjou Moumouni (2006) entre l'abondance des aliments et la saison des pluies. Les fréquences de distribution des aliments stockés varient en fonction des régions. Cette différence est liée à la taille des troupeaux qui est inégalement répartie dans les trois zones d'étude, d'où, la nécessité pour certains éleveurs de pratiquer la transhumance. Les études menées par Dehoux et Hounsou-Vê (1993) ont montré que l'organisation de la transhumance est non seulement liée aux besoins hydriques, nutritionnels et sanitaires du bétail, mais aussi, aux besoins socio-culturels des éleveurs. Cependant, malgré le surpâturage, les éleveurs du Menabe central (Madagascar) pensent que les lieux de pâture doivent rester fixes, autrement dit, que les itinéraires de pâture ne doivent pas changer (Solomalala et al., 2010). Toutefois, le choix des éleveurs pour des lieux de pâture fixes au Menabe central semble en contradiction avec la présence de surpâturage. Tout comme dans la présente étude, les troupeaux de bovins pâturent le plus souvent à proximité des points d'eaux et dans les 
savanes à dominance graminées (Solomalala et al., 2010).

\section{Suivi sanitaire des animaux}

Les cas de pathologies ou symptômes de pathologies rencontrés sont multiples avec des proportions qui diffèrent en fonction des départements. Il est important de souligner que les éleveurs n'arrivent pas à faire le lien entre les symptômes et les pathologies. Toutefois, parmi ces symptômes, on peut citer la diarrhée, le piétin, les avortements, la toux, la gale, les lésions de stomatite, la fièvre, les jetages, le pica, le larmoiement, les plaies, l'amaigrissement et la météorisation, etc. Selon Dehoux et Hounsou-Vê (1993), les principales pathologies rencontrées dans les élevages de bovins sont : la trypanosomose, la brucellose bovine, la streptothricose, la tuberculose, la pasteurellose, la péripneumonie contagieuse bovine, la fièvre aphteuse, les parasitoses gastro-intestinales, la fasciolose bovine et le piétin. Avec une prévalence sérologique de $84 \%$ dans les différentes races bovines, les trypanosomoses animales constituent une des pathologies dominantes des bovins dans les deux départements (Doko et al., 2010). D'autres pathologies comme la brucellose bovine, caractérisée par des avortements et des inflammations au niveau des articulations, existent dans ces localités et doivent être dépistées par des diagnostics sérologiques (Adéhan et Youssao, 2002 ; Kouthinhouin et al., 2003 ; Kouamo et al., 2010). Pour ces maladies abortives, l'insémination artificielle peut être utilisée pour la prévention (Kouamo et al., 2009).

La totalité des éleveurs des trois Départements fait traiter les animaux par la médecine moderne. En plus, la plupart des éleveurs du Borgou et de l'Alibori font appel à la pharmacopée pour assurer le bien être des bovins. Ce mode de traitement par les plantes est aussi rapporté par Yacoubou (2011) dans la Commune de Sinendé et Alkoiret et al. (2009) sur les bovins, qui ont montré que les fermiers font usage des plantes locales pour la prévention et le traitement de certaines pathologies. Les trypanocides sont souvent administrés aux animaux dans les trois Départements. L'utilisation fréquente de ces trypanocides est liée au taux de prévalence élevé de cette pathologie. Doko et al. (2010) ont déterminé un taux de prévalence cumulé de $30 \%$, ce qui veut dire que 9 veaux sur 30 ont connu leur primo-infection avant douze mois.

\section{Suivi des animaux}

Le suivi des animaux est assuré par le propriétaire des animaux, sa femme, ses enfants, et ses employés dans les trois régions en plein temps ou à temps partiel, et chaque membre de la famille a une part de responsabilité dans le suivi et la conduite des élevages dans le Nord-Bénin. Ce mode de suivi est en adéquation avec le système d'élevage extensif traditionnel et surtout familial adopté en milieu paysan (Koutinhouin et al., 2009). Des résultats similaires ont été enregistrés par Yacoubou (2011) lors du suivi sanitaire des bovins de race Borgou dans les mêmes régions.

\section{Commercialisation des animaux}

De manière générale, les prix de vente obtenus lors de la présente étude sont supérieurs à ceux de Dehoux et Housounvê (1993) qui rapportent que les vaches sont vendues à $35000 \mathrm{~F} \mathrm{CFA}$, les taureaux à 50000F CFA et les taurillons 20000F CFA dans les élevages traditionnels du Nord Bénin. La différence entre ces prix et ceux obtenus au cours de notre étude peut s'expliquer par la cherté de la vie. La quantité moyenne journalière de lait produite par éleveur est corrélée avec la taille moyenne des troupeaux et à l'objectif de production. Des tendances similaires ont été rapportées par Koutinhouin et al. (2010) dans le Département de l'Atacora.

\section{Conclusion}

Il ressort de l'analyse des données de l'enquête sur la typologie des élevages de bovins de race Borgou dans les élevages traditionnels de la partie septentrionale du 
Bénin que les différences qui existent entre les personnes enquêtées sont parfois liées aux régions de résidence. Les éleveurs rencontrés au cours de notre étude sont majoritairement Peulh. Ils pratiquent pour la plupart la religion musulmane et sont non scolarisés. Les femmes éleveurs de bovins ne se retrouvent que dans le Borgou et sont très minoritaires. Les activités pratiquées dans la zone d'étude se caractérisent par l'association ElevageAgriculture. Les fréquences d'abreuvement sont liées à la répartition inégale des cours d'eau dans la zone d'étude. L'abreuvement est uniquement assuré par les points d'eau naturels. L'alimentation des animaux est assurée dans les trois régions par le fourrage en pâturage naturel, des résidus de récolte et le sel de cuisine. A l'issue de cette étude, trois types d'élevages ont été retenus. Le premier est composé d'éleveurs professionnels ou d'agriculteurs qui font le plus souvent un élevage de prestige. Le second type d'élevage est composé d'agro-éleveurs qui exploitent les animaux et leurs produits. Le type 3 concerne les éleveurs de bovins qui ont le commerce comme activité principale et qui font de l'embouche bovine dans la ville de Parakou. Dans le souci d'améliorer la productivité des élevages dans chaque type d'élevage, les propositions d'amélioration sont formulées. Pour les trois types d'élevage, il faut procéder à la scolarisation ou à l'alphabétisation des éleveurs et surtout de leurs enfants. Ces éleveurs sont pour la plupart des Peulh et par conséquent, le programme d'alphabétisation prôné par le gouvernement béninois doit se concrétiser en ciblant ces éleveurs Peulh. Les objectifs de production doivent être revus et clairement définis. Dans les élevages de type 1, il est nécessaire de sensibiliser les éleveurs sur les manques à gagner d'un élevage de prestige, de leur donner quelques notions sur l'exploitation judicieuse des animaux. Chez les éleveurs de type 2, il faut renforcer les capacités des éleveurs dans l'exploitation des animaux et les organiser en coopérative, diffuser auprès des éleveurs de ce type d'élevage des techniques d'alimentation des jeunes taurillons élevés en embouche herbagère sur pâturage naturel. Quant aux éleveurs de type 3, il serait nécessaire de les former sur les techniques d'engraissement des animaux et d'identifier les circuits d'approvisionnement des veaux à engraisser et de commercialisation des animaux.

\section{REFERENCES}

Adam SK, Boko M. 1993. Le climat du Bénin. In le Bénin. Edition SodimasEdicef: Paris; 96.

Adamou-N'Diaye AM, Ogodja OJ, Gbangboche AB, Adjovi A, Hanzen C. 2001. Intervalle de vêlage chez la vache Borgou au Bénin. Ann. Méd. Vét., 145: 130-136.

Adehan R, Youssao AKI. 2002. Epizotiologie des mycoplasmoses pulmonaires des ruminants domestiques au Bénin. Revue Méd. Vét., 153(6): 415-418.

Adjou Moumouni PF. 2006. Evaluation des performances zootechniques des bovins de race Borgou en sélection à la ferme d'élevage de l'Okpara, Bénin. Thèse de Doctorat en médecine vétérinaire, Ecole Inter-Etats des Sciences et Médecine Vétérinaire de Dakar (E.I.S.M.V.), Dakar, $\mathrm{N}^{\circ} 20$, p. 121.

Alkoiret IT, Awohouedji DYG, Akossou AYJ, Bosma RH. 2009. Typologie des systèmes d'élevage bovin de la commune de Gogounou au Nord-Est du Bénin. Ann. Sci. Agro. Bénin, 2(12): 77-98.

Alkoiret TI, Ggangboche AB. 2005. Fécondité de la race lagunaire au Bénin. Age au premier vêlage et intervalle entre vêlages. Revue Elev. Méd. Vét. Pays Trop., 58(12): 61-68.

Bani Kogui S. 2011. Gestion de l'alimentation des bovins de race Borgou: cas des Communes de Sinendé et de OuassaPéhunco. Mémoire de fin de formation pour l'obtention du diplôme de Licence Professionnelle en Production Animale, Ecole Polytechnique d'Abomey-Calavi, p.58.

Bierschenk T, Forster R. 2004. L'organisation sociale des peulh dans l'Est de l'Atacora (République du Bénin, Commune de 
Kouandé, Pehonco et Kérou). Gutemberg University, Arbeitspapiere / Working papers (46), Mainz, p.94.

Dehoux J-P, Hounsou-Ve G. 1993. Productibilité de la race bovine Borgou selon les systèmes d'élevage traditionnels au Nord-Est du Bénin. World Anim. Review, 74/75: 36-48.

Djenontin JA, Amidou M, Baco NM. 2004. Diagnostic gestion du troupeau: gestion des ressources pastorales dans les départements de l'Alibori et du Borgou au nord du Bénin. Bul. Rec. Agr. Bénin, 43: $30-45$.

Doko AS, Farougou S, Salifou S, Ehilé E, Geerts S. 2010. Dynamique des infections trypanosomiennes chez des bovins Borgou à la ferme de l'Okpara au Bénin. Tropicultura, 28(1): 37-43.

Domingo AM. 1976. Contribution à l'étude de la population bovine des états du golfe du Bénin. Thèse de Doctorat en médecine vétérinaire. Ecole Inter-Etats des Sciences et Médecine Vétérinaire de Dakar (E.I.S.M.V.), Dakar, p.148.

CeRPA. 2009. (Centre Régional pour la Promotion Agricole), Rapport annuel d'activités. Cotonou, 108p.

FAO. 2004. Banque de données, FAOTSTAT : Agriculture. Adresse URL: http//apps.fao.org/page/collection ?suset= agriculture $=\mathrm{fr}$. Consulté le 27 juillet 2010.

Houessionon FJB. 2011. Typologie des élevages de poulets locaux de l'espèce Gallus gallus en aviculture familiale au Bénin. Mémoire de fin de formation pour l'obtention du diplôme de Master en Production et Santé Animales, Ecole Polytechnique d'Abomey-Calavi, p.98.

Kouamo J, Habimana S, Alambedji Bada R, Sawadogo GJ, Ouedraogo GA. 2010. Séroprévalences de la brucellose, de la BVD et de l'IBR et impact sur la reproduction des femelles zébus Gobra et croisements inséminés en milieu traditionnel dans la région de Thiès au Sénégal. Ann. Méd. Vét., 161(7): 314321 .
Kouamo J, Sow A, Leye A, Sawadogo GJ, Ouedraogo GA. 2009. Amélioration des performances de production et de reproduction des bovins par l'utilisation de l'insémination artificielle en Afrique Subsaharienne et au Sénégal en particulier : état des lieux et perspectives. RASPA, 7(3-4): 139-148.

Koutinhouin B, Youssao AKI, Houehou AE, Agbadje PM. 2003. Prévalence de la brucellose bovine dans les élevages traditionnels encadrés par le Projet pour le Développement de l'Elevage (PDE) au Bénin. Revue Méd. Vét., 154(4): 271-276.

Koutinhouin GB, Youassao AKI, Tobada P, Kpodekon TM, Adimatin V. 2009. Influence de l'indice de température et d'humidité relative de l'air sur la fécondité de la vache Borgou élevée selon deux modes d'élevage au Bénin. Int. J. Biol. Chem. Sci., 3(6): 1336-1345.

Koutinhouin GB, Youssao AKI, Kpodekon TM, Gantoli YG. 2010. Influence de la traite précoce des vaches sur la croissance pondérale et l'état sanitaire des veaux en élevage traditionnel: cas de la zone périurbaine de Natitingou (Bénin). LRRD, 22 (02) 2010, http://www.lrrd.org/ lrrd21/02/kout22024.htm_MAEP. 2007.

Ministère de l'Agriculture, de l'Elevage et de la Pêche. 2007. Programme de relance des productions animales au Bénin. Cotonou, p. 205.

Perrot C, Landais E. 1993. Exploitations agricoles: pourquoi poursuivre la recherche sur les méthodes typologiques? Dossier: méthodes d'étude en milieu paysan. Cab. Rech. Dév., 33:13-23.

Roybin D. 1987.Typologie de fonctionnement d'exploitations : quelles applications pour le développement GIS Alpes du Nord /SUACI Montagne Alpes du Nord/INRA SAD/CGER Haute-Savoie, France, p. 45.

SAS. 1996. SAS/STAT User's guide, vers, 6, 4th ed, Cary, NC,USA, SAS Inst, 1996.

Solomalala NR, Ratsimbazafy A, Ramohavelo CD, Sorg J-P, Buttler A. 2010. Optimisation socio-économique et 
écologique des systèmes d'élevage (Menabe central, Madagascar). Sécheresse, 21(3): 211-218.

Somda J, Kamuanga M, Mendes A, Gomes J. 2004. Caractéristiques socioéconomiques et performances économiques des élevages laitiers en Guinée Bissau : cas des régions de Bafata et Gabu. Socio-economic Research Working Paper No 4, ITC (International Trypanotolerance Centre), Banjul, The Gambia, p. 48.

Yacoubou AS. 2011. Suivi sanitaire des bovins de race Borgou: cas des Communes de Sinendé et de OuassaPéhunco. Mémoire de fin de formation pour l'obtention du diplôme de Licence Professionnelle en Production Animale, Ecole Polytechnique d'Abomey-Calavi, p. 55.

Youssao AKI, Ahissou A, Toure Z. 2000a. Productivité de la race Borgou à la ferme d'élevage de l'Okpara au Bénin. Revue Elev. Méd. Vét. Pays Trop., 53(1): 67-74.
Youssao AKI, Ahissou A, Michaux C, Famir $\mathrm{F}$, Toure Z, Idrissou N-D, Leroy PL. 2000b. Facteurs non génétiques influençant le poids et la croissance de veaux de race Borgou à la ferme d'élevage de l'Okpara au Bénin. Revue Elev. Méd. Vét. Pays Trop., 53(3): 285292.

Youssao AKI, Koutinhouin GB, Kpodekon TM, Agnandjo H, Touré Z, Ahissou A. 2009. Influence d'une sélection phénotypique sur les performances de croissance et les caractères de développements musculaire et squelettique de jeunes bovins de race à la Ferme d'Elevage de l'Okpara (Bénin). Ann. Méd. Vét., 153: 105-111.

Youssao AKI, Koutinhouin GB, Kpodekon TM, Agnandjo H, Touré Z, Ahissou A, Renand G. 2007. Variabilité génétique des performances de croissance et des mesures corporelles de jeunes bovins de race Borgou à la Ferme d'Elevage de l'Okpara. RASPA, 5(3-4): 157-165. 